\title{
Project 1640 Observations of Brown Dwarf GJ 758 B: Near-infrared Spectrum and Atmospheric Modeling
}

\author{
R. Nilsson ${ }^{1,2,3}$, A. Veicht ${ }^{1}$, P. A. Giorla Godfrey ${ }^{1,4,5}$, E. L. Rice ${ }^{1,4,5}$, J. Aguilar ${ }^{6}$, L. Pueyo ${ }^{7}$, L. C. Roberts, Jr. ${ }^{8}$, R. Oppenheimer ${ }^{1}$, \\ D. Brenner ${ }^{1}$, S. H. Luszcz-Cook ${ }^{1}$, E. Bacchus ${ }^{9}$, C. Beichman ${ }^{1,10}$, R. Burruss ${ }^{8}$, E. Cady ${ }^{8}$, R. Dekany ${ }^{11}$, R. Fergus ${ }^{12}$, L. Hillenbrand ${ }^{3}$, \\ S. Hinkley ${ }^{13}$, D. King ${ }^{9}$, T. Lockhart ${ }^{8}$, I. R. Parry ${ }^{9}$, A. Sivaramakrishnan ${ }^{7}$, R. Soummer ${ }^{7}$, G. Vasisht ${ }^{8}$, C. Zhai ${ }^{8}$, and \\ N. T. Zimmerman ${ }^{7}$ \\ ${ }^{1}$ Astrophysics Department, American Museum of Natural History, Central Park West at 79th Street, New York, NY 10024, USA; rnilsson@amnh.org \\ ${ }_{2}$ Department of Astronomy, Stockholm University, AlbaNova University Center, Roslagstullsbacken 21, SE-106 91 Stockholm, Sweden \\ ${ }^{3}$ Department of Astronomy, California Institute of Technology, 1200 E. California Boulevard, MC 249-17, Pasadena, CA 91125, USA \\ ${ }^{4}$ Physics Program, The Graduate Center, City University of New York, New York, NY 10016, USA \\ ${ }^{5}$ Department of Engineering Science \& Physics, College of Staten Island, 2800 Victory Boulevard, Staten Island, NY 10314, USA \\ ${ }^{6}$ Department of Physics and Astronomy, The Johns Hopkins University, Baltimore, MD 21218, USA \\ Space Telescope Science Institute, 3700 San Martin Drive, Baltimore, MD 21218, USA \\ 8 Jet Propulsion Laboratory, California Institute of Technology, 4800 Oak Grove Drive, Pasadena CA 91109, USA \\ ${ }^{9}$ Institute of Astronomy, Cambridge University, Madingley Road, Cambridge CB3 OHA, UK \\ ${ }^{10}$ NASA Exoplanet Science Institute, California Institute of Technology, Pasadena, CA 91125, USA \\ ${ }^{11}$ Caltech Optical Observatories, California Institute of Technology, Pasadena, CA 91125, USA
${ }^{12}$ Department of Computer Science, Courant Institute of Mathematical Sciences, New York University, 715 Broadway, New York, NY 10003, USA \\ ${ }^{13}$ School of Physics, University of Exeter, Stocker Road, Exeter, EX4 4QL, UK \\ Received 2015 July 26; revised 2017 February 18; accepted 2017 February 28; published 2017 March 24
}

\begin{abstract}
The nearby Sun-like star GJ 758 hosts a cold substellar companion, GJ 758 B, at a projected separation of $\lesssim 30$ au, previously detected in high-contrast multi-band photometric observations. In order to better constrain the companion's physical characteristics, we acquired the first low-resolution $(R \sim 50)$ near-infrared spectrum of it using the high-contrast hyperspectral imaging instrument Project 1640 on Palomar Observatory's $5 \mathrm{~m}$ Hale telescope. We obtained simultaneous images in 32 wavelength channels covering the $Y, J$, and $H$ bands $(\sim 952-1770 \mathrm{~nm})$, and used data processing techniques based on principal component analysis to efficiently subtract chromatic background speckle-noise. GJ 758 B was detected in four epochs during 2013 and 2014. Basic astrometric measurements confirm its apparent northwest trajectory relative to the primary star, with no clear signs of orbital curvature. Spectra of SpeX/IRTF observed T dwarfs were compared to the combined spectrum of GJ $758 \mathrm{~B}$, with $\chi^{2}$ minimization suggesting a best fit for spectral type T7.0 \pm 1.0 , but with a shallow minimum over T5-T8. Fitting of synthetic spectra from the BT-Settl13 model atmospheres gives an effective temperature $T_{\text {eff }}=741 \pm 25 \mathrm{~K}$ and surface gravity $\log g=4.3 \pm 0.5 \mathrm{dex}(\mathrm{cgs})$. Our derived best-fit spectral type and effective temperature from modeling of the low-resolution spectrum suggest a slightly earlier and hotter companion than previous findings from photometric data, but do not rule out current results, and confirm GJ $758 \mathrm{~B}$ as one of the coolest sub-stellar companions to a Sun-like star to date.
\end{abstract}

Key words: brown dwarfs - instrumentation: adaptive optics - instrumentation: spectrographs - planets and satellites: detection - stars: individual (GJ 758) - techniques: high angular resolution

\section{Introduction}

Techniques for high-contrast imaging have now matured to a level where direct detections of substellar companions to nearby stars are becoming frequent. Adaptive optics combined with coronagraphy, and sophisticated software speckle-reduction techniques using angular differential imaging and spectral differential imaging, have revealed low mass-ratio companions at 10-100 au around A stars (Lagrange et al. 2010; Marois et al. 2010; Rameau et al. 2013), and high mass-ratio companions at wider separations around later type stars (Lafrenière et al. 2008; Currie et al. 2014). ${ }^{14}$ Distinguishing between different formation scenarios, primarily core accretion (e.g., Pollack et al. 1996) or gravitational instability (e.g., Boss 2011), requires tight constraints on the orbital and physical parameters of these systems. It should be noted that

\footnotetext{
${ }^{14}$ This should not be taken to imply that high mass-ratio companions to early type stars do not exist, as recently shown in aperture masking interferometry observations by Hinkley et al. (2015).
}

direct imaging is still restricted to relatively large separations (beyond tens of au) and high mass $\left(>M_{\text {Jup }}\right.$ ) and/or young companions.

Out of the current two dozen or so directly imaged substellar companions, some have still only been observed in thermal emission in a few broad near-infrared (near-IR) photometric bands. However, instruments like Project 1640 (P1640; Oppenheimer et al. 2012) at the Palomar Hale telescope, Gemini Planet Imager (Macintosh et al. 2014) at Gemini South, and SPHERE (Beuzit et al. 2006) at the Very Large Telescope, are now able to simultaneously image and obtain low-resolution spectra using chromatic speckle suppression, allowing improved atmospheric characterization of gas-giant exoplanets and companion BDs.

GJ 758 (HIP 95319, HD 182488) is a Sun-like (spectral type G8 V) star located 15.76 pc away (van Leeuwen 2007) that has a substellar companion (GJ $758 \mathrm{~B}$ ) at a projected separation of $\sim 30 \mathrm{au}$, detected in $H$ band Subaru/HiCIAO imaging by Thalmann et al. (2009), confirmed in $L^{\prime}$ band MMT/Clio 
imaging by Currie et al. (2010), and followed up by multi-band ( $J, H, K_{c}, L^{\prime}, M$, and narrow band $C H 4 S$ and $C H 4 L$ filters) Subaru/HiCIAO, Gemini/NIRI, and Keck/NIRC2 imaging by Janson et al. (2011). ${ }^{15}$ Models presented in those papers have suggested an effective surface temperature of $\sim 600 \mathrm{~K}$, making it a T8-T9 dwarf, the coldest imaged companion of a Sun-like star, and one of the most important "planet-like" objects accessible for detailed study due to its proximity. The derived companion mass depends chiefly on its age, which, using any current method of age determination for main-sequence stars, remains highly uncertain. A wide range of possible ages, from 0.7-8.7 Gyr, have been derived, suggesting a companion mass ranging from $10 M_{\text {Jup }}$ (exoplanet region) to $40 M_{\text {Jup }}$ (BD region). While Takeda et al. (2007) derived an age of $0.7 \mathrm{Gyr}$ based on isochronal fits, Valenti \& Fischer (2005) and Holmberg et al. (2009) suggested several Gyr using the same method. Together with a non-detection of lithium in the atmosphere of GJ 758, Thalmann et al. (2009) decided to exclude the lower age in favor of activity and rotation based ages of 5-9 Gyr (Barnes 2007; Mamajek \& Hillenbrand 2008; Thalmann et al. 2009), narrowing the estimated mass range to 30-40 $M_{\text {Jup. }}$.

Although spectra have been obtained of many field BDs, including late $\mathrm{T}$ dwarfs, their even less constrained ages and metallicities make them hard to confidently model. Tightly constraining the physical properties of $\mathrm{T}$ dwarf companions around Sun-like stars will greatly contribute to our understanding of BDs in general, and place them in relation to planets and planetary system formation scenarios.

In this paper we present high-contrast imaging observations of GJ 758, using Hale/P1640, where we obtain the first lowresolution near-IR spectrum of the $\mathrm{B}$ companion, at four epochs, and model the companion's temperature, surface gravity, and spectral type. We also confirm the presence of methane in its atmosphere, as previously suggested from multiband photometry (Janson et al. 2011). A follow-up paper (J.Aguilar et al. 2017, in preparation) will present more detailed astrometric analysis and orbital simulations, using our new detection epochs and previous observations.

\section{Observations}

Palomar Observatory's $5.1 \mathrm{~m}$ Hale telescope is equipped with PALM-3000 (P3k; Dekany et al. 2013), a woofer-tweeter AO system with a 3388 actuator high-order deformable mirror (DM) and a 241 actuator low-order DM, which was used in conjunction with the P1640 instrument (Oppenheimer et al. 2012). P3k can, with recent hardware and software upgrades, reach Strehl ratios of $92 \%$ in the $K$ band $(100 \mathrm{~nm} \mathrm{rms}$ wavefront error, corresponding to $0.86 \%, 0.76 \%, 0.68 \%$ Strehl-ratios in $H, J$, and $Y$, respectively), on bright stars and in good seeing conditions (Burruss et al. 2014, and R. Burruss 2016, private communication). P1640 combines an apodized pupil Lyot coronagraph (Hinkley 2009; Soummer et al. 2009) with an internal calibration system (CAL; Zhai et al. 2012; Cady et al. 2013; Vasisht et al. 2014) for wavefront sensing and correction of residual phase and amplitude distortions at the occulter, and an integral-field spectrograph with a $200 \times 200$ lenslet array sampling the focal plane. Details on the instrument can be found in Oppenheimer et al. (2012) and Hinkley et al.

\footnotetext{
${ }^{15}$ During the referee process of this paper, additional results from observations with VLT/SPHERE were published by Vigan et al. (2016).
}

(2011). The covered wavelength range is $969-1797 \mathrm{~nm}$, encompassing the near-IR $Y, J$, and $H$ bands in 32 channels, at a spectral resolution of $\Delta \lambda=26.7 \mathrm{~nm}$. The total field-ofview (FOV) is approximately 3 !" $8 \times 3$ !" 8 .

GJ 758 was observed at an airmass of 1.00-1.05 at five different occasions, ${ }^{16}$ from 2012-06 to 2014-09, as presented in Table 1. Observing conditions varied, with the 2013-10 data taken at $\sim 1$ !' 1 seeing (in $V$ band), while 2012-06 data were obtained at a seeing far above the P1640 limiting $\sim 1$ "! 35 value for specified performance, and the 2014-06 data suffering from significant "mirror seeing" from large temperature gradients above the telescope main mirror. After pointing the telescope to the star, and making an initial AO tune-up, we used an internal white-light source to iterate on low- and high-order wavefront corrections with CAL. Final rms wavefront errors were on the order of $10 \mathrm{~nm}$. Reference astrometric spots, for locating the stellar center behind the occulting mask (Marois et al. 2006; Sivaramakrishnan \& Oppenheimer 2006), were introduced by applying a sinusoidal pattern on the DM. At each observing occasion, we obtained 3-10 long (3-6 minutes), occulted exposures, as well as 3-10 short (1.5-3.0 s), unocculted, "core" exposures, of the star. We also observed spectral standard stars for spectral calibration, and binary standards for plate-scale and position angle (PA) calibration (see Section 3).

\section{Data Reduction}

Descriptions of the overall P1640 data reduction procedure, as well as details of most individual pipeline modules, have been presented elsewhere (see, e.g., Oppenheimer et al. 2013), but for completeness we summarize the steps below.

\subsection{Cube Extraction}

The raw images, consisting of nearly 40,000 tightly packed spectra, each covering roughly $32 \times 3$ pixels on the detector, are extracted and converted into data cubes (measuring $250 \times 250 \times 32$ in $x \times y \times \lambda$, where $x$ and $y$ are the number of pixels in the first and second dimension, and $\lambda$ is the number of wavelength channels in the third dimension) by the $P 1640$ Data Cube Extraction Pipeline (PCXP; Zimmerman et al. 2011). PCXP uses the location of spectra (dots) from laser exposures at 1310 and $1550 \mathrm{~nm}$, together with sky flats, to create a focal plane solution that maps individual spectra to lenslets and corresponding boxel positions. Wavelength channels in which telluric atmospheric water absorption lines are strong (mainly channels 8, and 17-18) display some degree of cross-talk from adjacent spectra, and so does the first and last channel where our sensitivity falls steeply. Estimated errors at those wavelengths are consequently much larger than in the rest of the spectrum. Although those channels could have been omitted in the final analysis, we include them with corresponding errors in Section 5 since trimming the spectra did not significantly change the results.

\subsection{Dispersion Correction}

Using the four astrometric reference spots, we determine the star's centroid position in each wavelength channel, and track both atmospheric and instrumental dispersion shifts through the

\footnotetext{
${ }^{16}$ UT dates are given as year-month, year-month-day (in compliance with ISO 8601) throughout the paper, and as Julian days and Besselian years in Table 2 .
} 
Table 1

Observations of GJ 758 with Project 1640

\begin{tabular}{lcccc}
\hline \hline $\begin{array}{l}\text { Date } \\
\text { (UT) }\end{array}$ & $\begin{array}{c}\text { Julian Date } \\
\text { (days) }\end{array}$ & $\begin{array}{c}N_{\exp } \times t_{\text {exp }} \\
(\mathrm{s})\end{array}$ & $\begin{array}{c}\text { Estimated Seeing }^{\text {a }} \\
(\text { (") }\end{array}$ & $\begin{array}{c}\text { rms-WFE }^{\mathrm{b}} \\
(\mathrm{nm})\end{array}$ \\
\hline $2012-06-17$ & 2456095.8840277782 & $3 \times 366.6$ & 1.5 & $\ldots$ \\
$2013-07-21$ & 2456494.8199421302 & $10 \times 185.9$ & 1.1 & 7.5 \\
$2013-10-18$ & 2456583.5734722228 & $5 \times 185.9,6 \times 278.8$ & $1.0^{\mathrm{c}}$ & \\
$2014-06-10$ & 2456818.9438657411 & $7 \times 371.8$ & 4.5 & \\
$2014-09-07$ & 2456907.6952893524 & $8 \times 371.8$ & 9.0 & \\
\hline
\end{tabular}

Notes.

${ }^{\text {a }}$ Mean FWHM of PSF in $V$ band as recorded by Palomar 18 inch seeing monitor observing Polaris. Not recorded on 2013-07-21.

${ }^{\mathrm{b}}$ High-order wavefront error from final CAL iteration. Not recorded on 2012-06-17.

${ }^{c}$ Effective seeing was closer to 2". 0 due to high temperature gradient, with the telescope mirror being a few degrees warmer than the outside air.

cube. The image slices are then shifted in $x$ and $y$ with sub-pixel accuracy to align and center the star at $(x, y)=(126,126)$ in each cube (the cubes are later padded with a 251 st row and column of zeros to place the center of the star in the center pixel). As the radial position of reference spots and speckle noise is wavelength dependent, we can also derive a radial scaling relation, scale each image, and use cross-correlation for even finer sub-pixel image registration. This is all handled in the Cube Alignment Centering and Stacking (CACS, R. Nilsson et al. 2017, in preparation) pipeline module, which gives a final image registration accuracy of $\sim 0.2$ pixel $(1-\sigma$ deviation), and saves a hypercube $\left(x \times y \times \lambda \times N_{\exp }\right.$, where $N_{\text {exp }}$ is the number of exposures) for our speckle suppression algorithms. Aperture photometry of the reference spots also allow us to determine the $\lambda$ location of two telluric water absorption bands in our spectra (at 1100 and $1380 \mathrm{~nm}$ ), which together with knowledge of the instrument filter bandpass edges can be used to derive the shift and stretch function that aligns the cubes in the wavelength dimension.

\subsection{Speckle Suppression}

The wavelength-dependent speckle noise was modeled and subtracted using both KLIP (Soummer et al. 2012) and S4 (Fergus et al. 2014), to check for consistency. Both algorithms employ principal component analysis (PCA), but with component decomposition performed in different dimensions. Karheunen-Loeve Image Projection is used to decompose the speckle noise pattern into its principal components (PCs) by projecting companion-free regions of an image onto a Karheunen-Loeve basis. The algorithms then use the most prominent set of PCs to forward model speckle noise in the image to remove flux associated with the speckles but not with a putative companion.

\subsection{Point-source Identification}

Speckle reduced (residual) images in each channel and cube were filtered by convolving them with a model point-spread function (PSF) core to reduce noise, producing a "detection map" in which we could search for companion signals (Fergus et al. 2014). We also produced signal-to-noise ( $\mathrm{S} / \mathrm{N}$ ) maps by local calculation of the noise in each area of the residual image, using two different methods: (1) noise calculation in concentric annuli around the star (as normally done in high-contrast coronagraphic images with radially decreasing noise profiles), and (2) calculating the standard deviation of count levels in $20 \times 20$ pixel boxes around each pixel, with the inner $11 \times 11$ pixels left out. With the moving-box method we get an estimate of the increased noise along the astrometric spot trails, in regions that are cut out in the first method in order not to suppress overall $\mathrm{S} / \mathrm{N}$. The difference in calculated $\mathrm{S} / \mathrm{N}$ between the two methods turns out to be less than $10 \%$ in areas outside the spot trails. Significant peaks were found by $\sigma$ clipping and searching for regional maxima. Detected peaks were weighted by $\mathrm{S} / \mathrm{N}$ and summed up for each location, producing a list of the locations with the strongest peaks. At these locations we extracted raw mean spectra to compare with a range of reasonable companion spectra, and to exclude locations with a raw spectrum dominated only by bright peaks in the noisy water absorption channels. We also excluded peaks along the radial paths of the four astrometric reference spots.

\subsection{Spectral Extraction}

After locating the centroid pixel of a detected point-source in the residual cubes, we run S4's spectral extraction code, which again performs speckle-suppression on the aligned and centered hypercube, but this time with local optimization in the area where the suspected companion is located. By varying the model parameters (such as the size of test zone, $\Delta \theta$ and $\Delta r$, and the number of PCs, $N_{\mathrm{PC}}$, used), and measuring the change of the extracted candidate companion spectrum, as well as of a number of (typically 50) background points and fake source insertions at the same radius, the parameters that minimize the noise and most faithfully retrieve the fake source spectrum can be determined. This ensures that the speckle noise is optimally modeled and subtracted, without attenuating the companion flux by overfitting the data. A brief justification of chosen optimal S4 spectral extraction parameters is presented in Section 4.3. The results agree with the more rigorous treatment demonstrated in A. Veicht et al. (2017, in preparation).

Note that in the spectral extraction we also simultaneously fit both the speckle and companion models to the observed data, and the spectrum of the companion model is jointly estimated along with the PCA coefficients of the companion model. The $\mathrm{S} / \mathrm{N}$ of an extracted companion spectrum will thus in general be higher than for the original detection signal. The total gain in $\mathrm{S} / \mathrm{N}$ from the full optimization procedure is about a factor of two in $J$ and $H$ compared to the initial detection image. Also note that the errors in the extracted companion spectrum are derived from the extracted fake insertions at the same projected radius, corresponding to the standard method of $\mathrm{S} / \mathrm{N}$ determination in high-contrast imaging. More information about the S4 and KLIP spectral extractions procedure can be found in the appendix of Oppenheimer et al. (2013), and a 

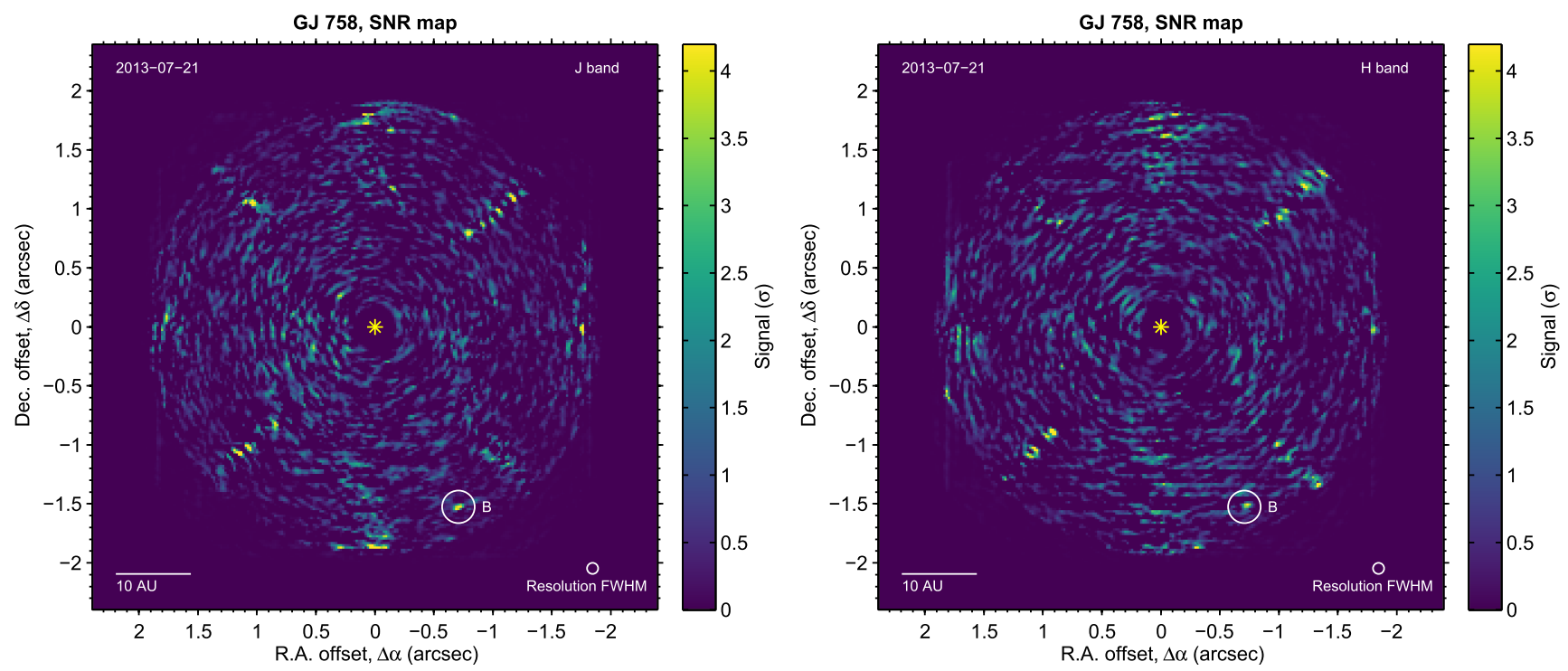

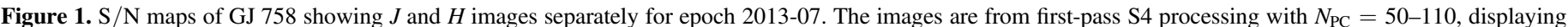

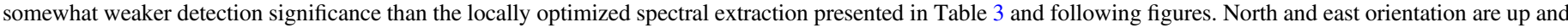

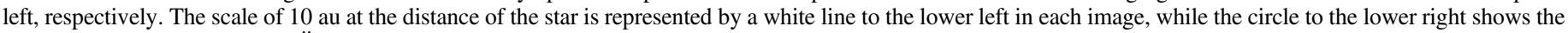
spatial resolution, FWHM =0."0958, measured from the PSFs of multiple core exposures. Both maps use the same linear color scale.

detailed analysis of S4's spectral extraction stability and error estimation is given in A. Veicht et al. (2017, in preparation).

\subsubsection{Spectral Calibration}

Each night, we obtained unocculted exposures of several calibrator stars of well-known spectral types with spectra available in the IRTF catalog of IR spectral standards (Cushing et al. 2008; Rayner et al. 2009). They were observed just before or after the occulted GJ 758 exposures, at similar airmass. Although core exposures of the G8 V primary could in principle have been used, they all saturated the detector in our shortest exposure setting. Calibrator PSF cores were fitted and shifted on a sub-pixel level to correct for atmospheric and instrument induced dispersion, and center the stars through the image cube. Their integrated count levels per wavelength channel, $S_{\text {cal }}\left(\lambda_{\text {P1640) }}\right)$ were found using aperture photometry, with a circular aperture enclosing the outermost visible Airy ring, and surrounding annulus for background calculation and subtraction. The IRTF template spectrum, $S_{\text {temp }}\left(\lambda_{\text {IRTF }}\right)$, corresponding to the spectral type of the observed calibrator, was degraded to P1640 spectral resolution by convolving with a Gaussian having a full width at half maximum equal to the resolution of our instrument, and then rescaling to match the integrated flux in our wavelength range. The Spectral Response Function (SRF), which the extracted companion flux is divided with to correct for atmospheric transmission and instrument sensitivity, is given by the ratio of the extracted calibrator flux to the down-sampled template spectrum: $R\left(\lambda_{\mathrm{P} 1640}\right)=S_{\text {cal }}\left(\lambda_{\mathrm{P} 1640}\right) / S_{\text {temp }}\left(\lambda_{\mathrm{P} 1640}\right)$.

To validate the SRF used to correct each hypercube and determine the SRF airmass dependence, the chosen SRFs were compared to a sequence of calibrator exposures obtained over several observing runs. As an additional consistency check on the shape and wavelength solution of each SRF, we compared them with the SRFs derived from the astrometric grid spots in the GJ 758 occulted data. Photometry of the latter has to be multiplied by $\lambda^{2}$ due to the chromaticity of the spot-inducing DM ripple, but can otherwise be used for simultaneous spectral calibration (Sivaramakrishnan \& Oppenheimer 2006), assuming that they are bright enough for a high $\mathrm{S} / \mathrm{N}$, and that the star's infrared spectrum and/or spectral type is well known.

Our wavelength accuracy depends on the cube extraction with PCXP (Zimmerman et al. 2011), which uses the focalplane solution derived from sky flats and monochromatic light exposures at $1310 \mathrm{~nm}$ and $1150 \mathrm{~nm}$, and which can often be up to one channel off in either direction. However, as previously mentioned (see Section 3.2), anchoring and scaling the wavelength axis using the two telluric water absorption bands (at 1100.1 and $1380.1 \mathrm{~nm}$ ), and the filter edges, brings the accuracy down to below $\pm 26.4 \mathrm{~nm}$ (the width of a channel).

\section{Results}

In this section we present resulting, PCA optimized data products from the speckle-suppression code: residual hypercubes $\left(x \times y \times \lambda \times N_{\exp } \times N_{\text {PC }}\right)$ and extracted companion spectra. GJ $758 \mathrm{~B}$ is weakly detected in four out of five epochs (including one marginal detection, see Table 1). KLIP revealed the companion in data from 2013-10 and 2014-09, and was used to verify the results of S4 in those two epochs. Our main results are thus based on $\mathrm{S} 4$ produced data products, which are our primary focus below.

\subsection{Residual Images and S/N Maps}

Speckle-reduced image cubes were examined, both using the source detection method outlined in Section 3.4 and by eye, to locate potential companion peaks for spectral extraction. At the expected location of GJ $758 \mathrm{~B}$ (extrapolated from astrometry given in Janson et al. 2011) we find significant $(>3 \sigma)$ flux peaks in three epochs, and a marginal $(>2.5 \sigma)$ detection in one epoch (see Table 1 for detection levels), in multiple wavelength channels in the $J$ and $H$ bands. Although we are measuring some flux in $Y$, it is at low statistical significance and can only be considered a marginal detection. In Figure 1 we show $\mathrm{S} / \mathrm{N}$ maps for the $J$ and $H$ bands, separately, for the 2013-07-21 epoch, averaged over all exposures and over the number of PCs 

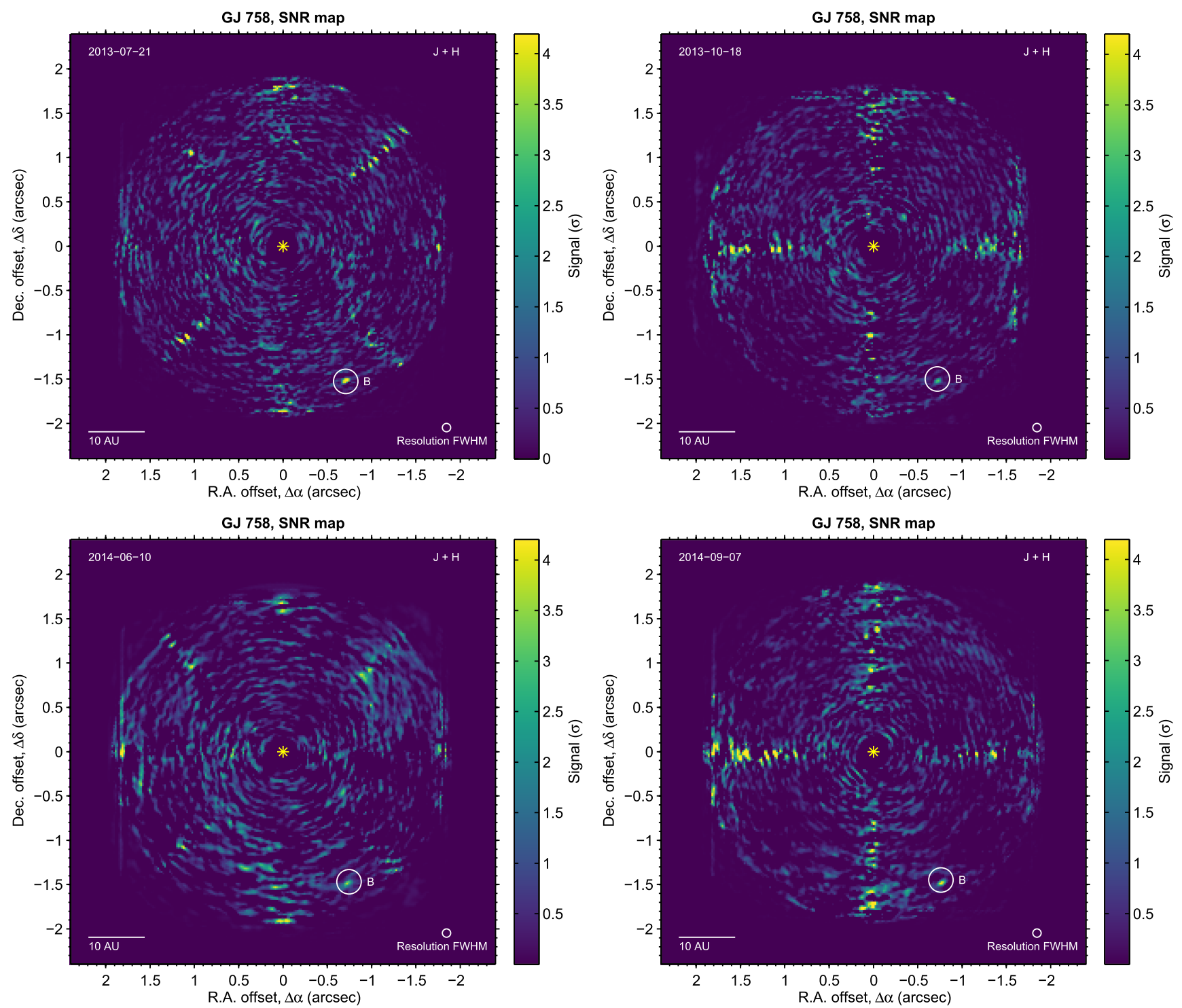

Figure 2. S/N maps of GJ 758 showing combined images from wavelength channels covering the $J$ and $H$ band for all four epochs. The images are from first-pass $\mathrm{S} 4$ processing with $N_{\mathrm{PC}}=50-110$

that give the highest $\mathrm{S} / \mathrm{N}$. Combined $J$ and $H \mathrm{~S} / \mathrm{N}$ maps for all four epochs are shown in Figure 2. Due to computational limitations, we ran $\mathrm{S} 4$ with a fixed $\Delta \theta=3$ pixels in the detection phase, which gave highest companion $\mathrm{S} / \mathrm{N}$ in $\mathrm{PC}$ range 50-110. Note that this is not the same optimal number of PCs as determined for the locally optimized spectral extraction (see Sections 4.3 and 4.4), which usually gives an optimum at $\Delta \theta=5$, and $N_{\mathrm{PC}}=100-250$.

Although several other peaks in individual $\mathrm{S} / \mathrm{N}$ maps seem to reach $>3-\sigma$ levels, all of them were discarded based on them either moving radially through consecutive wavelength channels (indicative of being a residual speckle), or being located along the trails of the four astrometric spots. Bright spot residuals are visible diagonally in the images from 2013-07 and 2014-06, and horizontally/vertically in images from 2013-10 and 2014-09 (Figure 2). We do not detect the faint background star previously found by Thalmann et al. (2009) and Janson et al. (2011) within our FOV. Due to the high proper motion of GJ 758, the star should have moved considerably to the southwest, but still remain within our FOV (see Section 4.2 and Figure 3). It is likely too faint to be seen in our images.
As the contrast in our observations could not be reliably calculated due to saturation of the primary in core exposures, we instead estimate reached contrast levels by comparing signal strengths to the absolute magnitudes of the companion (from Janson et al. 2011) and the primary. GJ 758 B has $M_{J}=17.58$ and $M_{H}=18.16$, and the parent star has $M_{J}=4.38$ and $M_{H}=3.76$. This indicates $3-\sigma$ contrasts of $2 \times 10^{-6}$ and $7 \times 10^{-7}$ in $J$ and $H$ respectively, reached at an angular separation of 1 !" $6-1$ !"7.

\subsection{Astrometry}

Determining the location of a companion relative its host star with high precision in coronagraphic data is notoriously difficult. First, finding the position of the stellar core in the focal plane, hidden behind the coronagraphic mask, poses challenges. Second, we have to measure the precise location of a faint point-source signal in an image littered with residual speckles. For IFUs in particular, there are additional concerns when calibrating the field-distortion, plate-scale, and detector orientation. 


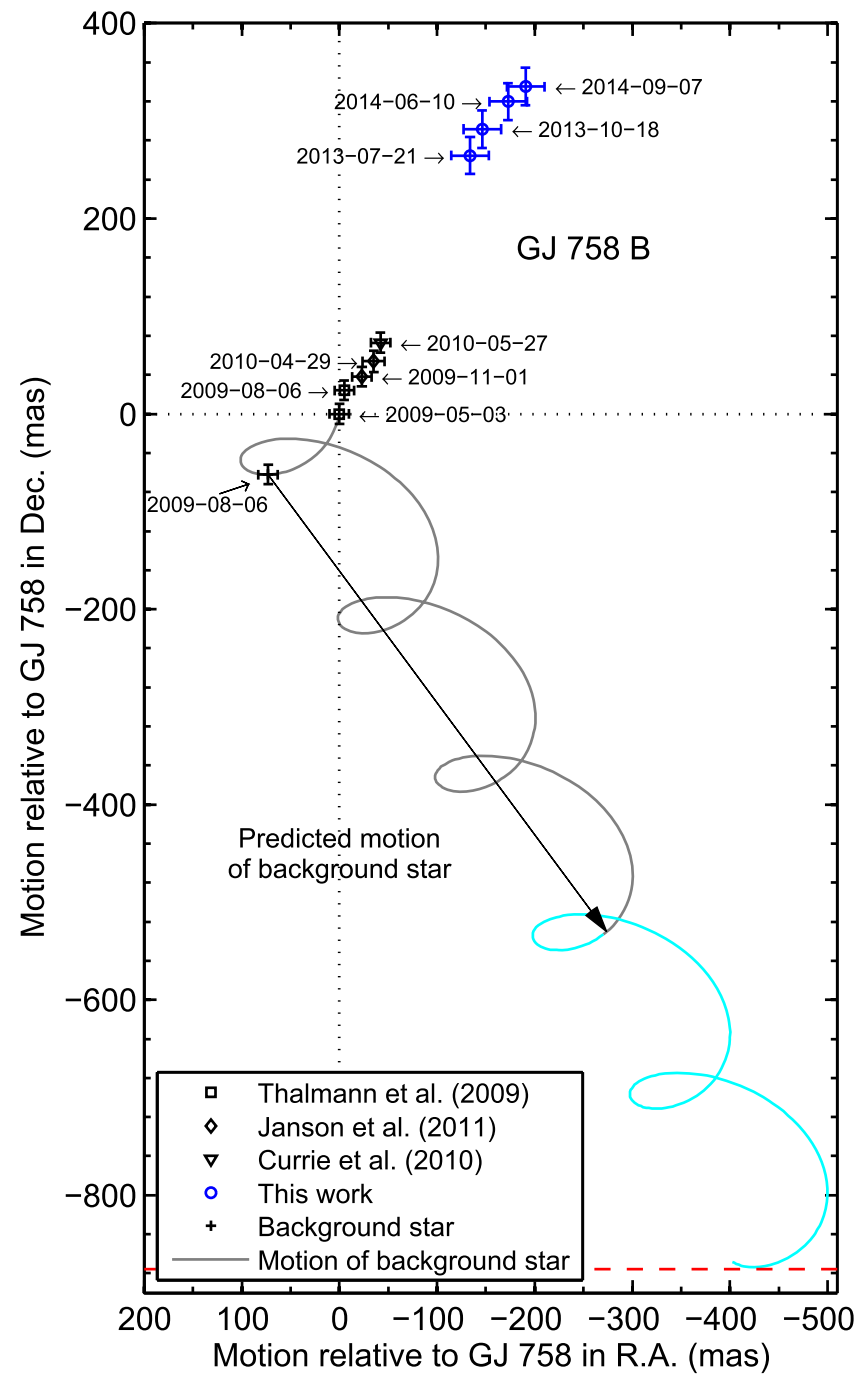

Figure 3. Astrometric analysis of GJ 758 B, showing its position and implied northwest motion relative to the primary, since its discovery in 2009-05. The black markers are from previous astrometry, and the blue markers are from the four epochs of detection presented in this work, all with associated error bars displayed. The predicted motion (due to GJ 758's proper motion and annual parallactic motion) of the background star discovered in 2009-08 is shown as a black curve, turning cyan at our first observing epoch in 2012-06. The star was in our FOV, nearing the edge of our detector (marked with a red dashed line) for the last two epochs, but too faint to be detected.

On each observing occasion, we observed a sample of binaries (HIP 72447 for 2013-07; HD 13594, HIP 34860 and HIP 97222 for 2013-10; HIP 88745 and HIP 107354 for 201406; and HIP 10403, HIP 12619, HD 3304, HD 11803, HIP 25826, HIP 97222, and HIP 88745 for 2014-09) chosen from the Sixth Catalog of Orbits of Visual Binary Stars (Hartkopf \& Mason 2011) to have well-determined orbital parameters. Several exposures of each binary were obtained, at different positions on the detector to examine effects of fielddistortion. The plate scale and absolute north orientation of the detector were found to be very stable, and we calculated values for the different epochs all agreeing to within 1- $\sigma$ errors. We used the derived mean plate scale, $19.16 \pm 0.18$ mas/pixel, and PA offset of $-72^{\circ} .43 \pm 1.06$ for all epochs. It should be noted that several of the binary calibrators lacked cataloged error estimates on their orbits, which means that the uncertainties on the plate scale and PA offset may be higher than stated.
From the processing of image cubes with CACS (Section 3.2) we expect the primary star to be aligned and centered in the images to a precision of $\sim 0.2$ pixels. A larger error on the astrometry comes from the isotropic Gaussian fit to the GJ $758 \mathrm{~B}$ peaks, which can be distorted by residual speckle noise. As a conservative precaution we present astrometric errors equivalent to \pm 1 pix in Figure 3 and Table 2 .

The measured angular separation and PA of GJ $758 \mathrm{~B}$ relative to the primary star is presented in Table 2, together with previous astrometry data. Figure 3 shows the motion of GJ $758 \mathrm{~B}$ with respect to the primary since the discovery by Thalmann et al. (2009) in 2009-05, and also includes the expected motion of a nearby faint object previously detected in three epochs (Thalmann et al. 2009; Janson et al. 2011) and determined to be a background star, but undetected in our observations. Because of GJ 758's high proper motion $\left(\mu_{\text {R.A. }}=83.40\right.$ mas yr $^{-1}$ and $\mu_{\text {decl. }}=162.32$ mas yr $^{-1}$; van Leeuwen 2007), the background star should have moved more than 600 mas to the southeast since its discovery in 2009-08 to our first observed epoch in 2012-06. In the time span of our five observed epochs, it would be expected to lie on the cyan colored curve in Figure 3, within the P1640 FOV with GJ 758 centered on the detector, but very close to our southern edge (marked by a dashed red line) for our two last epochs.

GJ $758 \mathrm{~B}$ is moving northwest as seen in the rest frame of GJ $758 \mathrm{~A}$, but so far with no discernible orbital curvature in nine epochs of astrometric data spanning almost 5.5 years. A linear extrapolation from previous astrometry gave a good prediction on the final measured positions of the companion. A forthcoming paper will discuss orbital simulations (J. Aguilar et al. 2017, in preparation).

The projected separation of the companion to the GJ 758 primary is $\sim 26 \mathrm{au}$ in our last (2014-09) epoch, using the parallax from the most recent Hipparcos reduction (van Leeuwen 2007).

\subsection{Optimization of S4 Speckle-suppression Parameters}

For spectral extractions, the width of the test region in polar coordinates is $\Delta \theta=5$ pixels (as opposed to the $\Delta \theta=3$ pixels used in the S4 detection phase). This has in previous tests of S4 given a better signal extraction than $\Delta \theta=3$ or 7 . Using the $1-\sigma$ errors derived from fake insertions as described in Section 3.5, we calculate the mean error over all wavelength channels in the normalized spectra, and plot them versus the number of PCs, $N_{\text {PC }}$, used in the PCA image reconstruction and spectral extraction. As can be seen in the left panel of Figure 4, a minimum is reached at $N_{\mathrm{PC}} \approx 160-225$ for the 2013-07 epoch. The typical range of optimal $N_{\mathrm{PC}}$ found in $\mathrm{S} 4$ spectral extraction is consistently between 100 and 250, depending on data quality. For the final spectra of each epoch, we calculated error-weighted mean spectra from $N_{\mathrm{PC}}=\{160,170,180,190$, $200,225\},\{190,200,225,250\}$, and $\{120,130,140,150,160$, $170,180,190\}$, for 2013-07, 2014-06, and 2014-09, respectively. For 2013-10 we did not obtain a clear minimum and decided to include the full $N_{\mathrm{PC}}=10-400$ range.

To show the total spread in extracted spectra over all tested number of PCs, $N_{\mathrm{PC}}=\{10,20,30, \ldots, 400\}$, we plot them together with the $N_{\text {PC }}$ optimized sub-sample for the 2013-07 extraction in the right panel of Figure 4. 
Table 2

Astrometry for GJ 758 B

\begin{tabular}{|c|c|c|c|c|}
\hline $\begin{array}{l}\text { Besselian Date } \\
\text { (years) }\end{array}$ & $\begin{array}{l}\text { Julian Date } \\
\text { (days) }\end{array}$ & $\begin{array}{c}\text { Position Angle, } \theta_{\mathrm{PA}} \\
\left({ }^{\circ}\right)\end{array}$ & $\begin{array}{c}\text { Angular Separation, } \rho \\
\left({ }^{\prime \prime}\right)\end{array}$ & Reference \\
\hline 2009.338 & 2454955.2 & $197.77 \pm 0.15$ & $1.879 \pm 0.010$ & Thalmann et al. (2009) \\
\hline 2009.598 & 2455050.1 & $198.18 \pm 0.15$ & $1.858 \pm 0.010$ & Thalmann et al. (2009) \\
\hline 2009.836 & 2455137.1 & $198.83 \pm 0.31$ & $1.850 \pm 0.010$ & Janson et al. (2011) \\
\hline 2010.326 & 2455316.0 & $199.34 \pm 0.34$ & $1.839 \pm 0.011$ & Janson et al. (2011) \\
\hline 2010.403 & 2455344.1 & $199.76 \pm 0.15$ & $1.823 \pm 0.015$ & Currie et al. (2010) \\
\hline 2012.461 & 2456095.9 & $204.92 \pm 0.31$ & $1.680 \pm 0.019$ & This work \\
\hline 2013.553 & 2456494.8 & $205.69 \pm 0.31$ & $1.661 \pm 0.019$ & This work \\
\hline 2014.441 & 2456818.9 & $206.95 \pm 0.29$ & $1.648 \pm 0.019$ & This work \\
\hline 2014.684 & 2456907.7 & $207.75 \pm 0.28$ & $1.643 \pm 0.019$ & This work \\
\hline
\end{tabular}

Table 3

S/N of GJ 758 B Detection in YJH After Locally Optimized Spectral Extraction

\begin{tabular}{lccccc}
\hline \hline $\begin{array}{l}\text { Date } \\
(\mathrm{UT})\end{array}$ & $\begin{array}{c}\text { Julian Date } \\
\text { (days) }\end{array}$ & $\begin{array}{c}\mathrm{S} / \mathrm{N}_{Y} \\
(\sigma)\end{array}$ & $\begin{array}{c}\mathrm{S} / \mathrm{N}_{J} \\
(\sigma)\end{array}$ & $\begin{array}{c}\mathrm{S} / \mathrm{N}_{H} \\
(\sigma)\end{array}$ & $\begin{array}{c}\mathrm{S}_{\mathbf{N}, \max }{ }^{\mathrm{a}} \\
(\sigma)\end{array}$ \\
\hline $2013-07-21$ & 2456494.8199421302 & 4.2 & 9.5 & 3.9 & 12.7 \\
$2013-10-18$ & 2456583.5734722228 & 1.7 & 4.7 & 3.0 & 6.4 \\
$2014-06-10$ & 2456818.9438657411 & 4.2 & 3.0 & 1.8 & 3.6 \\
$2014-09-07$ & 2456907.6952893524 & 4.5 & 7.7 & 3.2 & 7.7 \\
\hline \multicolumn{2}{c}{ Combined SRN for all epochs } & 7.9 & 14.1 & 7.3 & $\ldots$ \\
\hline
\end{tabular}

Note.

${ }^{a}$ Highest statistical significance of signal in any wavelength channel from locally optimized spectral extractions.

\subsection{Final GJ 758 B Spectrum}

Extracted final spectra from the four detected epochs are overplotted in Figure 5. Error bars represent 1- $\sigma$ deviations derived from fake source insertions and extractions, as explained in Section 3.5, using the weighted error over included optimal $N_{\mathrm{PC}}$ range (and $\Delta \theta=5$ pixels). As an example of the variation between extracted spectra for the explored $N_{\text {PC }}$ range, all extractions for the 2013-10 epoch are presented in Section 4.3. Parameter optimization for processing of the data from 2014-06 was difficult due to its bad quality (severe "mirror seeing"), thus the spectrum from that date has lower $\mathrm{S} / \mathrm{N}$, and may be considered only a marginal detection. Maximum S/Ns are listed as detection significance in Table 1. All spectra have been normalized by division with their mean flux density in the observed wavelength range. Channels in the photometric $Y$ band, covering 960-1080 nm, are very noisy due to our decreased sensitivity toward shorter wavelengths, with no significant detection of the companion in either epoch. The same holds true over $1350-1480 \mathrm{~nm}$, the shaded region in Figure 5, where we have bad sensitivity due to absorption from telluric atmospheric water vapor. That is also a region where have large uncertainties in the applied SRF since the spectral calibrators from which it is derived were observed at different times and thus in different conditions. The most striking features in all spectra are sharp peaks around 1280 and $1580 \mathrm{~nm}$ - a spectral signature characteristic of $\mathrm{T}$ dwarfs (see Section 5.2). Overall, the agreement between spectra in the $J(1014-1327 \mathrm{~nm})$ and $H(1477-1784 \mathrm{~nm})$ bands is good (within $2 \sigma$ ), and we use the combined spectrum (weighted average with weighted errors) for further modeling and analysis in the next section.

\subsubsection{KLIP Confirmation of S4 Spectral Extraction}

In addition to extracting the P1640 observed spectrum of GJ 758 B using S4, we independently confirmed the extraction using KLIP. The companion was immediately detected in KLIP reductions of observations on 2013-10-18 and 2014-09-07. Figure 6 shows how well the combined KLIP extractions from those two epochs match the combined S4 extractions from all four detected epochs. Additional modeling of the combined KLIP extracted spectra following the procedure in Section 5.2 confirms the inferred spectral type, effective temperature and surface gravity of the companion to within stated errors.

\section{Discussion}

This section contains further analysis of our results, presenting photometry, atmospheric modeling, and a prediction of GJ 758's RV trend.

\subsection{Photometry}

P1640's simultaneous coverage of the $Y, J$, and $H$ bands, gives it an advantage over many other high-contrast imaging instruments when it comes to relative flux calibration and the object properties derived from near-IR photometric colors. Assuming a well-characterized SRF, it essentially obtains automatic relative flux over all channels, without adding uncertainties from calibration of separate observations taken with different filters. Post-processing introduces additional uncertainties in the absolute flux, which can be quantified using fake insertions or $N_{\mathrm{PC}}$ plateaus. Despite this challenge, we can nevertheless estimate the $J-H$ color of GJ $758 \mathrm{~B}$. After multiplying with the Gemini/NIRI MKO filter transmission curves used in Janson et al. (2011), we integrate the normalized flux densities over $J$ and $H$ to obtain $J-H=-0.7 \pm 0.3 \mathrm{mag}$. This is consistent (within the errors) with the $J-H=-0.58 \pm 0.28$ mag derived by Janson et al. (2011), confirming its exceptionally blue near-IR color, similar to cool cloud-free field T dwarfs (Kirkpatrick et al. 2011; Dupuy \& Liu 2012). The small change in $J-H$ color along the emperical T dwarf sequence for field objects makes it hard to determine a more exact spectral type based on color alone. Our atmospheric analysis below does however indicate that GJ $758 \mathrm{~B}$ could possibly be a slightly warmer and earlier type BD than previously found. $^{17}$

\footnotetext{
${ }^{17}$ This is also consistent, depending on interpretation, with the SPHERE results presented by Vigan et al. (2016) during the referee processes of this paper.
} 

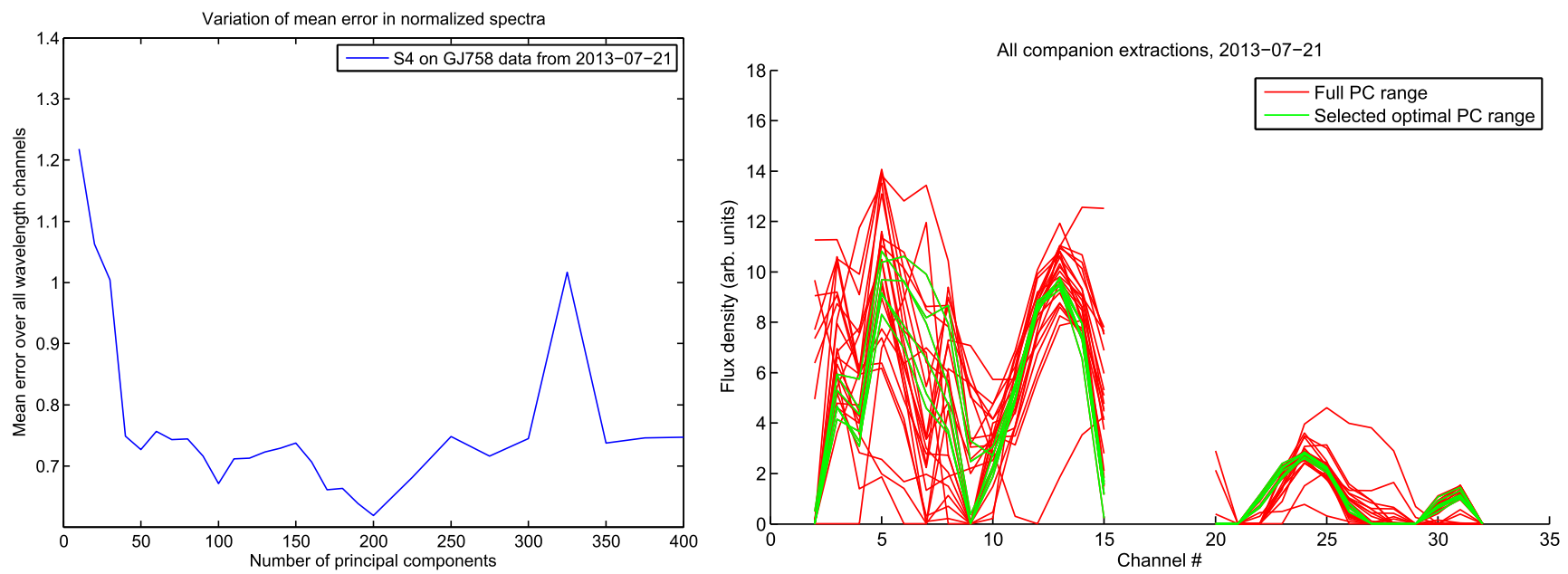

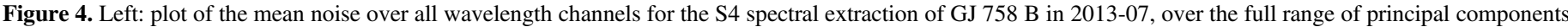

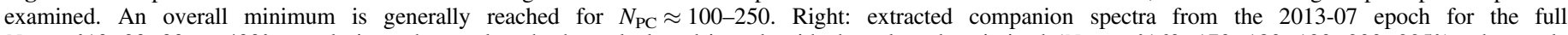

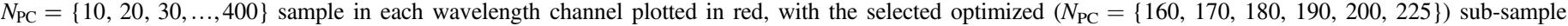
plotted in green.

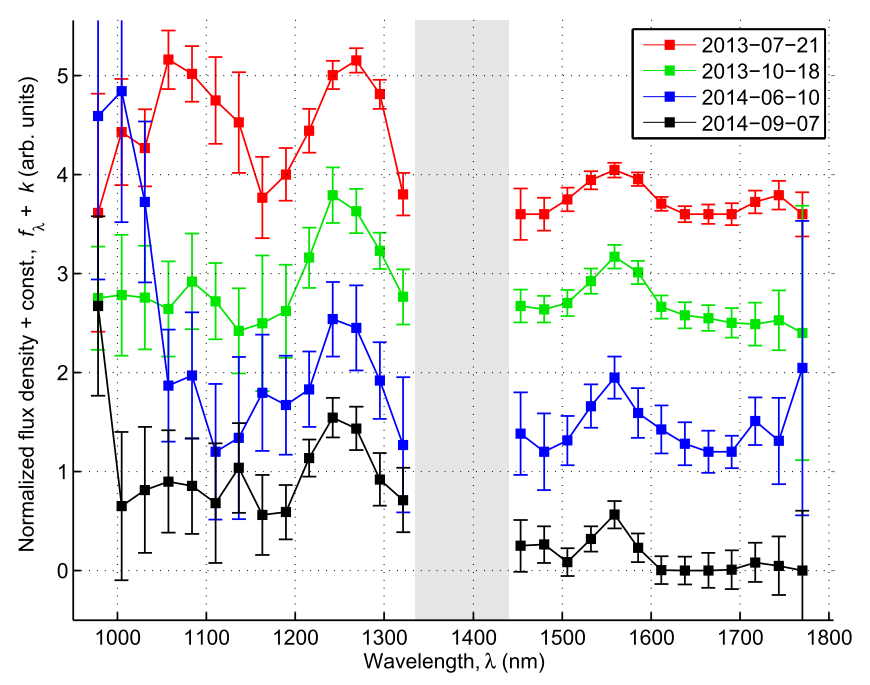

Figure 5. S4 extracted spectra of GJ 758 B from all four epochs in which it was detected, labeled with observing date as YYYY-MM-DD. Error bars show 1- $\sigma$ spectral deviations of 50 fake white-light sources inserted and extracted at the same projected angular distance from the primary star as the companion, averaged over the included $N_{\mathrm{PC}}$ range found from noise optimization (see Section 4.3).

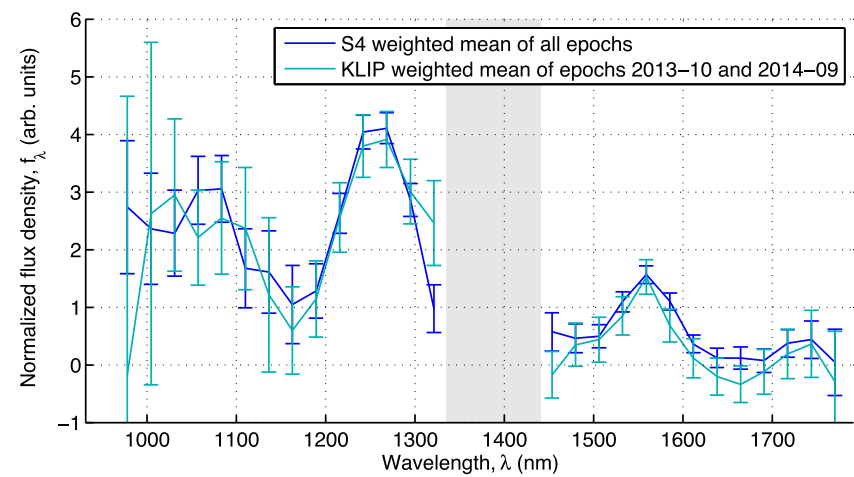

Figure 6. The KLIP extracted spectrum of GJ 758 B combined from the 2013-10-18 and 2014-09-07 observations, compared to the S4 extracted spectrum combined from 2013-07-21, 2013-10-18, 2014-06-10, and 2014-0907 data.

\subsection{Spectral Fitting}

Below we use both observed and synthetic spectra of Tdwarfs to perform spectral typing and derive physical characteristics of GJ $758 \mathrm{~B}$ based on the spectrum obtained with P1640. It is pertinent here to note that deriving any astrophysical object's effective temperature and surface gravity is inherently dependent on the model used and the data included, be it broad- or narrow-band photometry, lowresolution spectra, high-resolution spectra, different wavelength ranges, etc. The spectrophotometric fitting used here can give different answers than broader SED fitting, both of which can give different answers from atomic line ratios-all from the same model. In this sense, "temperature" is a somewhat philosophical value, with different methods and models producing systematically different numbers for the same object. Some authors have, for example, plotted spectra of these sorts of objects as brightness temperature versus wavelength to illustrate how each part of the spectrum arises from very different depths in the object's atmosphere (Matthews et al. 1996; Oppenheimer 1999).

\subsubsection{Empirical Analysis}

An effective way to estimate the spectral type of T dwarfs is by comparison of their near-IR $(0.8-2.5 \mu \mathrm{m})$ spectra to those of standard T dwarfs (Burgasser et al. 2006). Here, we compare the combined $Y J H$ GJ $758 \mathrm{~B}$ spectrum to 154 T dwarfs, ranging from spectral types $\mathrm{T} 0-\mathrm{T} 9$, including the T0-T8 standards, in order to estimate its spectral type. Most of the spectra were obtained from the SpeX Prism Library. ${ }^{18}$

The SpeX Prism spectra are binned to the resolution of the P1640 observation by adding the flux (and uncertainties in quadrature) within the wavelength bins appropriate to the lower resolution spectrum. A $\chi^{2}$ is calculated for each binned $\mathrm{T}$ dwarf spectrum compared to the GJ $758 \mathrm{~B}$ spectrum. We have removed the water band around $1.4 \mu \mathrm{m}$ from the fit, though fits were completed both with and without those flux points and the results were essentially identical. Errors from both the GJ $578 \mathrm{~B}$ spectrum and the binned T dwarf templates are used

${ }^{18}$ http://pono.ucsd.edu/adam/browndwarfs/spexprism/ 

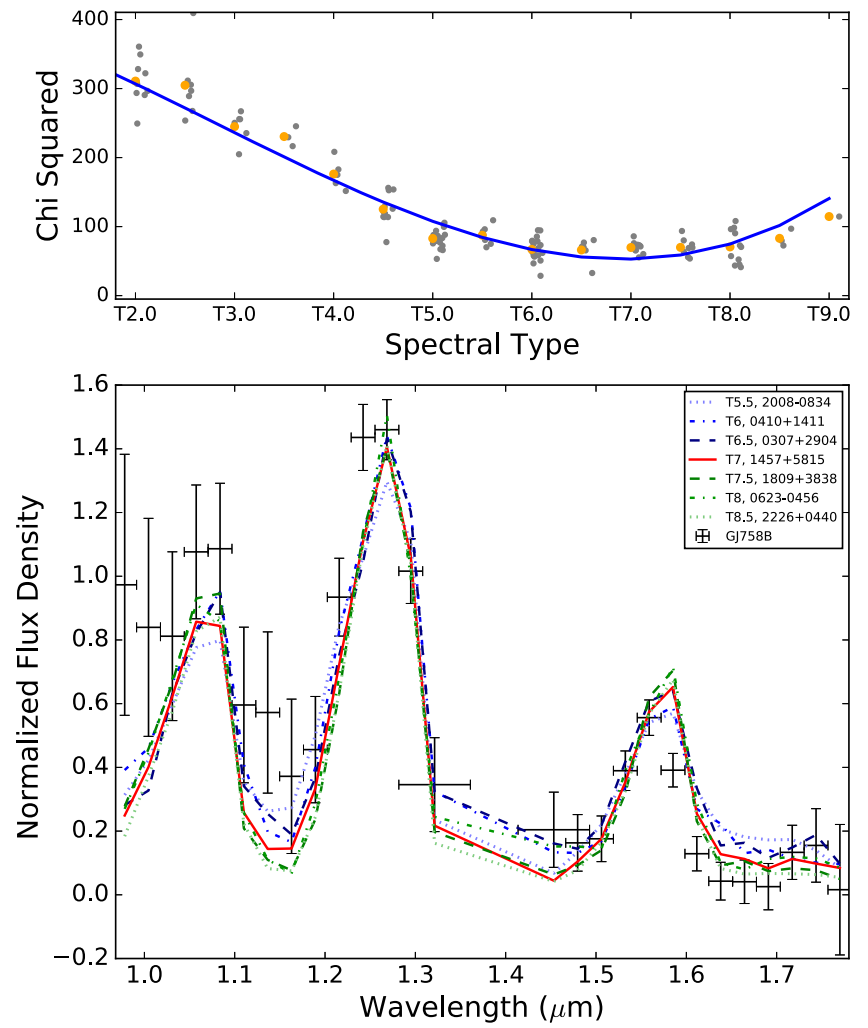

Figure 7. (Top) $\chi^{2}$ as a function of spectral type for T0-T9 objects. A thirdorder (blue) polynomial fit from T0-T9 based on the average $\chi^{2}$ results per spectral type, and the average $\chi^{2}$ points per spectral type (orange), are also shown. We derive a spectral type of T7.0 \pm 1 from this spectral comparison. (Bottom) P1640 spectrum of GJ 758 B (black crosses) plotted with binned and trimmed SpeX Prism spectra of T5.5-T8.5 objects.

in the calculation. Figure 7 (top) shows $\chi^{2}$ as a function of spectral type for each T dwarf template with a third order polynomial fit. Using the S4 extracted spectrum, a spectral type of T7.0 (WISE J145715.03+581510.2, Kirkpatrick et al. 2011) results in the minimum $\chi^{2}$ value of $\sim 60$ with 29 degrees of freedom. ${ }^{19}$ Figure 7 (bottom) shows this best fit, along with example fits, also of lowest $\chi^{2}$, for the surrounding Tdwarf spectral subtypes (WISE J200804.71083428.5, WISE J180901.07+383805.4, Mace et al. (2013); WISE J041054.48+141131.1, WISE J030724.59 +290447.4 , WISE J062309.94045624.6, WISE J222623.05 +044004.0 , Kirkpatrick et al. 2011). Based on these comparisons we estimate a spectral type of T7.0 \pm 1 for GJ $758 \mathrm{~B}$.

\subsubsection{Model Atmospheres}

T dwarfs are classified according to their near-IR spectra, but spectral types do not necessarily correspond directly or uniquely to physical properties (e.g., Kirkpatrick et al. 2008). To constrain atmospheric parameters, in this case effective temperature and surface gravity, we compare the observed P1640 spectrum of GJ 758 B to synthetic spectra from the BTSettl13 model atmospheres (Allard 2014). We use a grid of solar metallicity models with effective temperatures from $400-4500 \mathrm{~K}$ in increments of 50 or $100 \mathrm{~K}$ and surface gravities of $\log g=3.5,4.0,4.5,5.0$, and 5.5 (cgs units). The model fitting procedure is based on that described in Rice et al.

\footnotetext{
19 Note that our $\chi^{2}$ versus spectral type relation looks almost identical to the goodness-of-fit value versus spectral type plot in Figure 5 of Vigan et al (2016), also with a minimum of T7.0 after visual inspection.
}

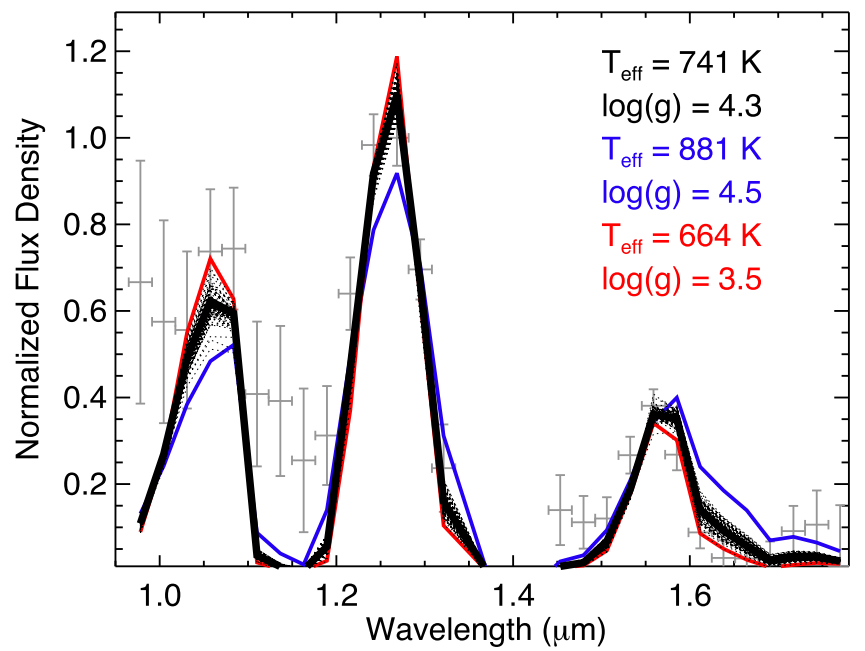

Figure 8. Best fit synthetic spectrum from BT-Settl13 models (black) using parameters derived from MCMC analysis of the complete $Y J H$ spectrum (excluding the terrestrial water absorption band) from S4 extractions of the P1640 spectrum of GJ 758 B (gray markers and error bars). 100 spectra were randomly chosen from the posterior distributions of the MCMC calculations to represent the range of model fits that are allowed within $1-\sigma$ uncertainty for the spectrum. The best fit parameters, $741 \mathrm{~K} / 4.3 \mathrm{dex}(\mathrm{cgs})$ for the $Y J H$ spectrum, are the $50 \%$ quantiles of the effective temperature and surface gravity parameters from the MCMC posterior distributions (see Figure 9. The BT-Settl13 spectra with parameters determined from $Y J$ and $H$ fits only are shown in red and blue, respectively.

(2015). Versions of the fitting procedure have been applied to P1640 spectra in Roberts et al. (2012), Hinkley et al. (2013), and Crepp et al. (2015). The fitting procedure is summarized briefly below.

Model spectra are binned from their native resolution of $\Delta \lambda=0.1 \mathrm{~nm}$ to match that of the $\mathrm{P} 1640(\Delta \lambda=26.4 \mathrm{~nm})$ spectrum. A goodness-of-fit parameter similar to $\chi^{2}$ (see Cushing et al. 2008) is calculated for each fit of the observed spectrum to each binned spectrum in the model grid. The model parameters for the spectrum with the minimum $\chi^{2}$ are used as the starting point for generating probability distributions, $P \propto \exp \chi^{2} / 2$, using a $10^{6}$-step Markov Chain Monte Carlo (MCMC) analysis using the Metropolis Hastings algorithm. The MCMC routine interpolates between calculated model spectra as it moves along the chain of steps. We find that jump sizes of $200 \mathrm{~K}$ in temperature and $2.0 \mathrm{dex}$ in surface gravity provide optimal acceptance ratios of $\sim 0.3-0.4$.

We fit model spectra to three versions of each extracted spectrum of GJ $758 \mathrm{~B}$ (and 100 random samples from the MCMC chain): (1) the complete $(Y J H)$ spectrum without the four flux points closest to the water absorption band at $1.4 \mu \mathrm{m}$, (2) the flux points blueward of $1.33 \mu \mathrm{m}(Y J)$, and the flux points redward of $1.45 \mu \mathrm{m}(H)$. Results for all three versions of the spectra from each extraction are presented in Figure 8) in black, red, and blue, respectively.

Posterior distributions from the subsequent MCMC analysis, presented in Figure 9, show a range in $T_{\text {eff }}$ from $\sim 620$ to $\sim 940 \mathrm{~K}$ for the three spectra. The posterior distributions marginalized over $\log g$ show a clear peak in $T_{\text {eff }}$ for the complete spectrum, with broader and slightly asymmetric histograms for $Y J$ and $H$ fits. The $\log g$ histograms cover a wide parameter range for the three fits, with the peak for $H$ fit falling below the edge of the model grid at low surface gravities. The peak at higher surface gravity values is higher and corresponds 


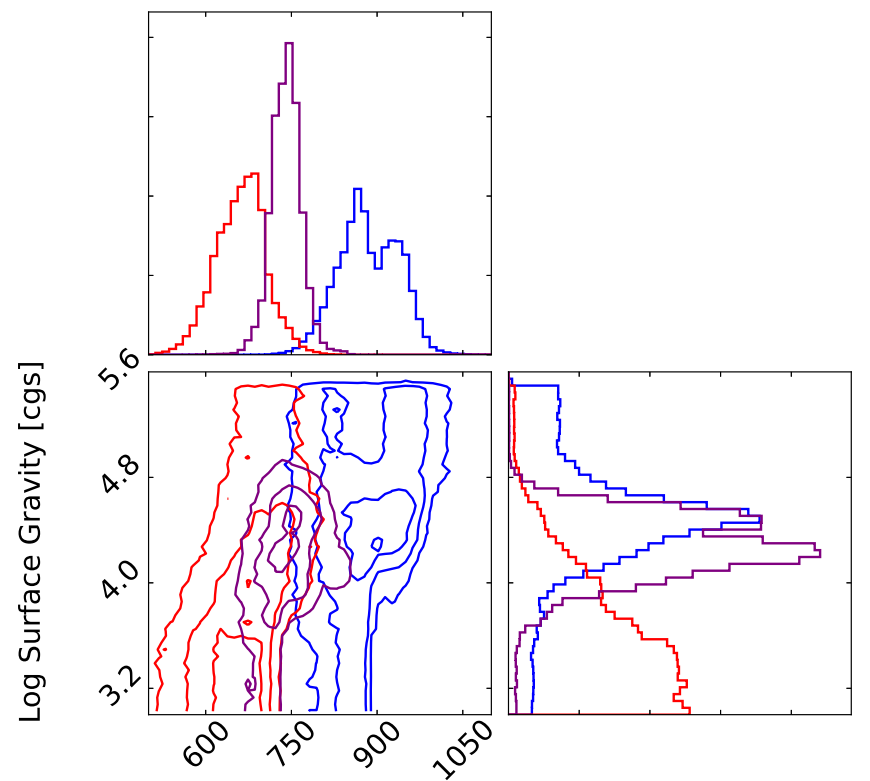

Effective Temperature (K)

Figure 9. Posterior distributions of the MCMC analysis using $10^{6}$ steps for the complete $Y J H$ spectrum (purple), $Y J$ (red), and $H$ (blue) S4 extracted spectrum of GJ $758 \mathrm{~B}$. Histograms show the distributions marginalized over gravity (top left) and temperature (bottom right). Model fits to the low-resolution nearinfrared spectrum from $\mathrm{P} 1640$ provide a better constraint in temperature than in surface gravity. GJ $758 \mathrm{~B}$ has a temperature of $T_{\text {eff }}=741 \pm 25 \mathrm{~K}$ and a surface gravity of $\log g=4.3 \pm 1.0 \mathrm{dex}(\mathrm{cgs})(1-\sigma$ uncertainties).

to slightly hotter $T_{\text {eff }}$ values. As was noted in Crepp et al. (2015) and Rice et al. (2015), we currently cannot reliably infer gravity from these low-resolution spectra. However, the higher end of the range is consistent with the gravity predicted by evolutionary models (e.g., Baraffe et al. 2003) based on the temperature of GJ $758 \mathrm{~B}$ and the age constraint provided by the primary star.

Using the mode posterior distributions and $68 \%$ confidence interval for the posterior of the spectral fits, we find that GJ $758 \mathrm{~B}$ has best-fit $T_{\text {eff }}$ and $\log g$ from the MCMC of $741 \pm 25 \mathrm{~K}$ and $4.3 \pm 0.5 \mathrm{dex}$ for $Y J H, 881 \pm 60 \mathrm{~K}$ and $4.5 \pm 0.5$ for $Y J$, and $664 \pm 45$ and 3.5 for $H$. The value for the complete $Y J H$ spectrum is consistent within the uncertainty to temperatures predicted by the spectral type $T_{\text {eff }}$ relationships of Filippazzo et al. (2015) for a T7.0 object $(825 \pm 113 \mathrm{~K}$ for the M6-T9 relationship).

The T7.0 \pm 1.0 spectral type we find from the spectral analysis of GJ $758 \mathrm{~B}$ is slightly earlier than the prediction of T8-T9 based on near- and mid-infrared photometry (Janson et al. 2011), as was the case for HD 19467 B (T5-T7 predicted from photometry, T5.5 \pm 1.0 from the P1640 spectral analysis Crepp et al. 2015). For the later spectral type and cooler temperature of GJ $758 \mathrm{~B}$, the surface gravity results are more consistent with predictions from evolutionary models $(\log g=3.0-5.0)$, but the broad MCMC posterior distributions show that model fits to low-resolution near-IR spectra are still unreliable for independent age confirmation (see also Rice et al. 2015). Based on COND03 evolutionary models (Baraffe et al. 2003) for the temperature of the companion and age of the primary star, we infer a mass of $40-50 M_{\text {Jup }}$ for GJ $758 \mathrm{~B}$. The derived properties of GJ $758 \mathrm{~B}$ are summarized in Table 4.

Although not included in our modeling, due to the difficulty and large uncertainties involved in deriving absolute fluxes
Table 4

Derived Properties for GJ 758 B

\begin{tabular}{ll}
\hline \hline Property & GJ 758 B \\
\hline Spectral type & T7.0 \pm 1 \\
Effective temperature, $T_{\text {eff }}$ & $741 \pm 25 \mathrm{~K}$ \\
Surface gravity, $\log g$ & $4.3 \pm 0.5$ dex $(\mathrm{cgs})$ \\
Mass, $M$ (inferred) & $40-50 M_{\text {Jup }}$ \\
\hline
\end{tabular}

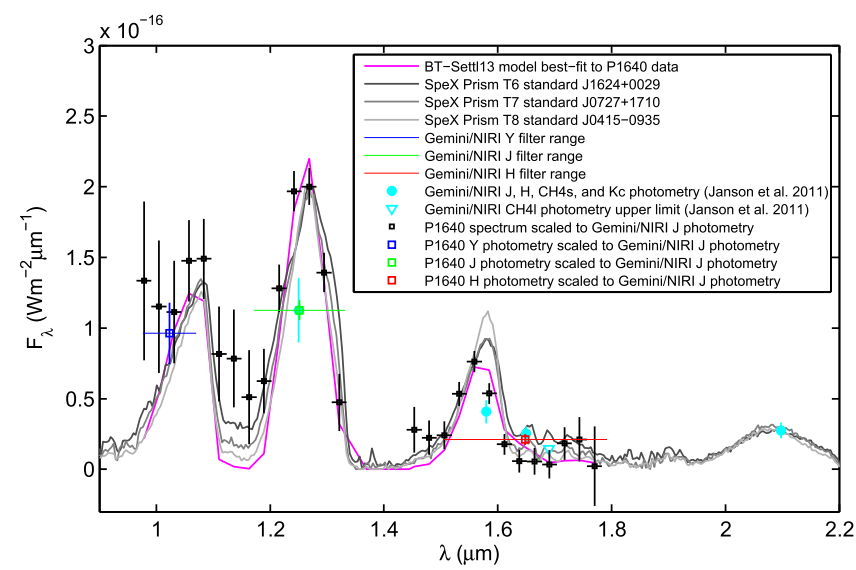

Figure 10. The obtained P1640 spectrum and derived $Y J H$ fluxes scaled by a factor found from matching Gemini/NIRI MKO $J$ band photometry with corresponding $J$ flux from P1640. Observed spectrum is shown in black, derived $Y J H$ fluxes in blue, green, and red, respectively (with thin horizontal bars showing the filter bandwidth). Previous photometry from Janson et al. (2011) is shown in cyan. The lines show the BT-Settl13 model and the SpeX Prism Library spectral type standards best-fit to the P1640 spectrum.

from this particular P1640 data set, we plot previous photometry from Janson et al. (2011) together with our data and spectral fits in Figure 10. The P1640 data have been scaled to the Gemini/NIRI $J$ band photometry, by calculating the corresponding P1640 flux from multiplication of the spectrum with the MKO $J$ filter transmission curve, integrating over the bandwidth, and matching the two flux densities. As can be seen in the figure, the resulting P1640 $\mathrm{H}$ band flux also essentially overlaps with that of Janson et al. (2011). The spectrum of GJ $758 \mathrm{~B}$ is not well-matched by other empirical data of similar objects, specifically T6-T8 SpeX Prism standards, especially in the $H$ band peak near $1.58 \mu \mathrm{m} . K_{c}$ data at $2.1 \mu \mathrm{m}$ does not seem to be a discriminator for spectral type, but such longer wavelength data (including $L^{\prime}$ and $M s$, Janson et al. 2011, not shown in figure) will be important in establishing $\log g$, $T_{\text {eff }},[\mathrm{Fe} / \mathrm{H}]$, and cloud cover from current (e.g., Morley et al. 2012; Saumon et al. 2012; Allard 2014) and future improved atmospheric models.

\subsubsection{Atmospheric Composition}

One of the main drivers for obtaining spectra of substellar companions to stars is the prospect of not only determining global characteristics, like $T_{\text {eff }}, \log g$, and $M_{*}$, but also say something about the chemical composition of their atmospheres. Multi-band photometry of GJ $758 \mathrm{~B}$ by Janson et al. (2011) suggested clear methane $\left(\mathrm{CH}_{4}\right)$ absorption, which is to be expected in Tdwarfs, with ever stronger absorption for cooler objects. Our spectrum clearly confirms the presence of $\mathrm{CH}_{4}$, with a deep absorption feature beyond $1600 \mathrm{~nm}$, and possibly absorption superimposed on $\mathrm{H}_{2} \mathrm{O}$ features at shorter wavelengths in our range. No other molecular species are 


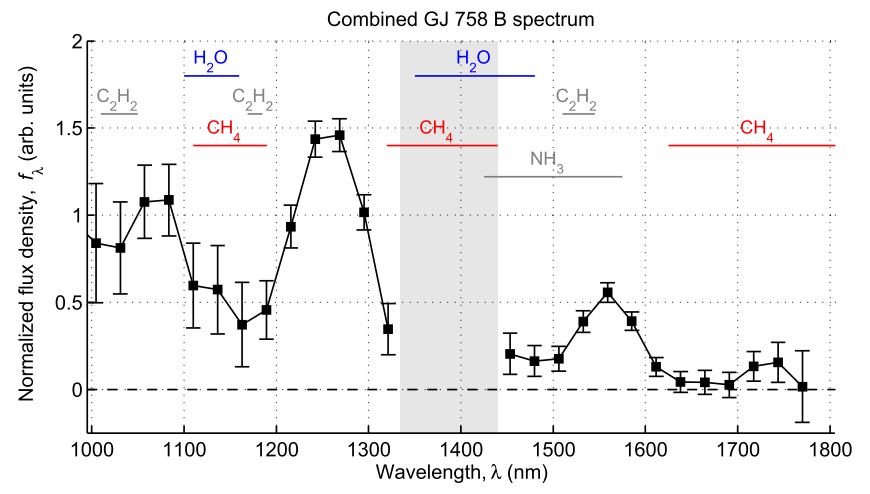

Figure 11. A normalized weighted average spectrum of GJ 758 B with weighted error bars, shown together with the expected location of molecular absorption bands in this wavelength range. There is clearly strong absorption of $\mathrm{CH}_{4}$ in the atmosphere, as expected for a cool $\mathrm{T}$ dwarf, while no identification of other molecular species is implied.

identified, but the location of the most prominent molecular absorption bands in the $\mathrm{P} 1640$ wavelength range $\left(\mathrm{NH}_{3}\right.$ and $\mathrm{C}_{2} \mathrm{H}_{2}$ ) are included for reference, and plotted together with the combined spectrum, calculated as the weighted average with mean errors, in Figure 11.

\subsection{Prediction of Radial Velocity (RV) Trend}

We use our new mass estimate of GJ 758 B, together with the $68 \%$ confidence bounds on the orbital parameters given in Janson et al. (2011), to make a rough estimate of the possibly observable RV trend of GJ 758. From Kepler's laws we find that the star's velocity in orbit around the system's barycenter can be approximated by:

$$
V_{*} \approx \frac{M}{M_{*}} \sqrt{\frac{G M_{*}}{a}} .
$$

For a companion mass $M$ in the range of $40-50 M_{\mathrm{Jup}}$, and orbital parameters (semimajor axis $a$, inclination $i$, and period $P$ ) from Table 2 in Janson et al. (2011), we get a maximum RV amplitude,

$$
V_{\mathrm{obs}}=V_{*} \sin i
$$

equal to $214 \mathrm{~m} \mathrm{~s}^{-1}$ for an orbital period of 170 years, and $53 \mathrm{~m} \mathrm{~s}^{-1}$ for a period of 843 years. Based on weighted median parameters, we obtain $132 \mathrm{~m} \mathrm{~s}^{-1}$ for a period of 299 years. This implies a change in observed RV of GJ 758 with between 2.52 and $0.13 \mathrm{~m} \mathrm{~s}^{-1}$ per year for the $68 \%$ confidence interval, or $0.88 \mathrm{~m} \mathrm{~s}^{-1}$ per year for median parameter values. These estimates will be further constrained with new orbital modeling using all nine astrometry data points in J. Aguilar et al. (2017, in preparation), but the above back-of-the-envelope calculation suggest that an RV trend could potentially be observable within a decade of continuous monitoring. However, to obtain model independent dynamical masses from orbit analysis with high accuracy we need both RV and astrometry with measurable curvature for a good part of the orbit. At that point, GJ $758 \mathrm{~B}$ could join HD 19467 (Crepp et al. 2014) as one of the coolest sub-stellar objects with dynamical mass and spectrum, making it an important benchmark object for brown dwarf studies.

\section{Conclusions}

We have detected GJ 758 B in four epochs, and obtained the first near-IR spectrum of this substellar companion to a Sunlike star. Based on atmospheric modeling we conclude the following:

1. The $Y J H$ spectrum of GJ $758 \mathrm{~B}$ is best fit with a spectral type T7.0 \pm 1.0 , an effective temperature $T_{\text {eff }}=741 \pm 25 \mathrm{~K}$ and surface gravity $\log g=4.3 \pm 0.5 \operatorname{dex}$ (cgs), but a slightly later spectral type and lower $T_{\text {eff }}$, compatible with results of Thalmann et al. (2009), Currie et al. (2010), and Janson et al. (2011), cannot be excluded.

2. A calculated $J-H$ color of $-0.7 \pm 0.3 \mathrm{mag}$ supports the found spectral type, in comparison to field brown dwarfs, but again does not constrain it to exclude later spectral types.

3. Combined with our derived effective temperature, evolutionary models suggest the companion has a mass $M=40-50 M_{\text {Jup }}$, for an assumed age of 5-9 Gyr.

4. Molecular absorption features in the spectrum of GJ $758 \mathrm{~B}$ confirm the presence of methane in its atmosphere.

5. A RV trend of the primary, GJ 758, due to gravitational interaction with the companion, is predicted to be observable within a decade of regular monitoring, which together with astrometry could allow a model-independent dynamical mass to be derived from orbit analysis, and possibly make GJ 758 B the coolest substellar object to be used as a standard point of reference for mass and spectrum determination of $\mathrm{T}$ dwarfs.

More detailed astrometric analysis is being performed, and will be applied to extensive modeling of GJ 758 B's orbital motion, in order to constrain its orbital parameters. This will be presented in J. Aguilar et al. (2017, in preparation).

We are grateful for the financial, scientific, and technical support that made this research possible: R.N. was funded by the Swedish Research Council's International Postdoctoral Grant No.637-2013-474. A portion of this work was supported by NASA Origins of the Solar System grant No. NMO7100830/ 102190, and NASA APRA grant No. 08-APRA08-0117. E.R. acknowledges support from the National Science Foundation under Grant No. 1211568 and NASA Astrophysics Data Analysis Program (ADAP) award 11-ADAP11-0169. J.A.'s work was facilitated in part by a National Physical Science Consortium Fellowship and by stipend support from the Laboratory for Physical Sciences in College Park, Maryland. Part of the research was carried out at the Jet Propulsion Laboratory, California Institute of Technology, under a contract with the National Aeronautics and Space Administration. We also thank the Palomar mountain crew, especially Bruce Baker, Mike Doyle, Carolyn Heffner, John Henning, Greg van Idsinga, Steve Kunsman, Dan McKenna, Jean Mueller, Kajsa Peffer, Paul Nied, Joel Pearman, Kevin Rykoski, Carolyn Heffner, Jamey Eriksen, and Pam Thompson.

Facilities: Palomar Observatory, Hale(P3k, P1640).

\section{References}

Allard, F. 2014, in Proc. IAU, Exploring the Formation and Evolution of Planetary Systems, ed. M. Booth, B. C. Matthews, \& J. R. Graham (Cambridge: Cambridge Univ. Press), 299

Baraffe, I., Chabrier, G., Barman, T., Allard, F., \& Hauschildt, P. H. 2003, A\&A, 402, 701 
Barnes, S. A. 2007, ApJ, 669, 1167

Beuzit, J.-L., Feldt, M., Dohlen, K., et al. 2006, Msngr, 125, 29

Boss, A. P. 2011, ApJ, 731, 74

Burgasser, A. J., Geballe, T. R., Leggett, S. K., Kirkpatrick, J. D., \& Golimowski, D. A. 2006, ApJ, 637, 1067

Burruss, R. S., Dekany, R. G., Roberts, J. E., et al. 2014, Proc. SPIE, 9148 , 914827

Cady, E., Baranec, C., Beichman, C., et al. 2013, Proc. SPIE, 8864, 88640K

Crepp, J. R., Johnson, J. A., Howard, A. W., et al. 2014, ApJ, 781, 29

Crepp, J. R., Rice, E. L., Veicht, A., et al. 2015, ApJL, 798, L43

Currie, T., Bailey, V., Fabrycky, D., et al. 2010, ApJL, 721, L177

Currie, T., Daemgen, S., Debes, J., et al. 2014, ApJL, 780, L30

Cushing, M. C., Marley, M. S., Saumon, D., et al. 2008, ApJ, 678, 1372

Dekany, R., Roberts, J., Burruss, R., et al. 2013, ApJ, 776, 130

Dupuy, T. J., \& Liu, M. C. 2012, ApJS, 201, 19

Fergus, R., Hogg, D. W., Oppenheimer, R., Brenner, D., \& Pueyo, L. 2014, ApJ, 794, 161

Filippazzo, J. C., Rice, E. L., Faherty, J., et al. 2015, ApJ, 810, 158

Hartkopf, W. I., \& Mason, B. D. 2011, AJ, 142, 56

Hinkley, S. 2009, PhD thesis, Columbia Univ.

Hinkley, S., Kraus, A. L., Ireland, M. J., et al. 2015, ApJL, 806, L9

Hinkley, S., Oppenheimer, B., Zimmerman, N., et al. 2011, PASP, 123, 74

Hinkley, S., Pueyo, L., Faherty, J. K., et al. 2013, ApJ, 779, 153

Holmberg, J., Nordström, B., \& Andersen, J. 2009, A\&A, 501, 941

Janson, M., Carson, J., Thalmann, C., et al. 2011, ApJ, 728, 85

Kirkpatrick, J. D., Cruz, K. L., Barman, T. S., et al. 2008, ApJ, 689, 1295

Kirkpatrick, J. D., Cushing, M. C., Gelino, C. R., et al. 2011, ApJS, 197, 19

Lafrenière, D., Jayawardhana, R., \& van Kerkwijk, M. H. 2008, ApJL, 689, L153

Lagrange, A.-M., Bonnefoy, M., Chauvin, G., et al. 2010, Sci, 329, 57

Mace, G. N., Davy Kirkpatrick, J., Cushing, M. C., et al. 2013, ApJS, 205, 6

Macintosh, B., Graham, J. R., Ingraham, P., et al. 2014, PNAS, 111, 12661
Mamajek, E., \& Hillenbrand, L. 2008, ApJ, 687, 1264

Marois, C., Lafreniere, D., Macintosh, B., \& Doyon, R. 2006, ApJ, 647, 612

Marois, C., Zuckerman, B., Konopacky, Q. M., Macintosh, B., \& Barman, T. 2010, Natur, 468, 1080

Matthews, K., Nakajima, T., Kulkarni, S. R., \& Oppenheimer, B. R. 1996, AJ, 112,1678

Morley, C. V., Fortney, J. J., Marley, M. S., et al. 2012, ApJ, 756, 172

Oppenheimer, B., Baranec, C., Beichman, C., et al. 2013, ApJ, 768, 24

Oppenheimer, B. R. 1999, PhD thesis, California Institute of Technology

Oppenheimer, B. R., Beichman, C., Brenner, D., et al. 2012, Proc. SPIE, 8447, 844720

Pollack, J., Hubickyj, O., Bodenheimer, P., et al. 1996, Icar, 124, 62

Rameau, J., Chauvin, G., Lagrange, A. M., et al. 2013, ApJL, 779, L26

Rayner, J. T., Cushing, M. C., \& Vacca, W. D. 2009, ApJS, 185, 289

Rice, E. L., Oppenheimer, R., Zimmerman, N., \& Roberts, L. C. 2015, PASP, 127, 479

Roberts, L. C., Rice, E. L., Beichman, C. A., et al. 2012, AJ, 144, 14

Saumon, D., Marley, M. S., Abel, M., Frommhold, L., \& Freedman, R. S. 2012, ApJ, 750, 74

Sivaramakrishnan, A., \& Oppenheimer, B. R. 2006, ApJ, 647, 620

Soummer, R., Pueyo, L., Ferrari, A., et al. 2009, ApJ, 695, 695

Soummer, R., Pueyo, L., \& Larkin, J. 2012, ApJL, 755, L28

Takeda, G., Ford, E. B., Sills, A., et al. 2007, ApJS, 168, 297

Thalmann, C., Carson, J., Janson, M., et al. 2009, ApJL, 707, L123

Valenti, J. A., \& Fischer, D. A. 2005, ApJS, 159, 141

van Leeuwen, F. 2007, A\&A, 474, 653

Vasisht, G., Cady, E., Zhai, C., Lockhart, T., \& Oppenheimer, B. 2014, Proc. SPIE, 9148, 914822

Vigan, A., Bonnefoy, M., Ginski, C., et al. 2016, A\&A, 587, A55

Zhai, C., Vasisht, G., Shao, M., et al. 2012, Proc. SPIE, 8447, 84476W

Zimmerman, N., Brenner, D., Oppenheimer, B., et al. 2011, PASP, 123, 746 